\title{
Correction to: Methods Used to Make Lipid Nanoparticles to Deliver LNA Gapmers Against IncRNAs into Acute Myeloid Leukemia (AML) Blasts
}

\author{
Chun-Tien Kuo, Robert J. Lee, and Ramiro Garzon
}

Correction to: Chapter 11 in: Alfons Navarro (ed.), Long Non-Coding RNAs in Cancer: Methods and Protocols, Methods in Molecular Biology, vol. 2348, https://doi.org/10.1007/978-1-0716-1581-2_11

In the original version of this book, Chapter 11 was published with incorrect author name and affiliation. It has now been updated in the revised version of this book.

The updated online version of this chapter can be found at:

https://doi.org/10.1007/978-1-0716-1581-2_11 